\title{
BIRC3 mutations in chronic lymphocytic leukemia - uncommon and unfavorable
}

\section{Eugen Tausch and Stephan Stilgenbauer}

Department of Internal Medicine III, Ulm University, Ulm, Germany

E-mail: STEPHAN STILGENBAUER - stephan.stilgenbauer@uniklinik-ulm.de

doi:10.3324/haematol.2019.238691

C hronic lymphocytic leukemia (CLL) is characterized by recurrent genomic aberrations as well as gene mutations, and BIRC3 (Baculoviral IAP Repeat Containing 3, also called cIAP2) can be affected by both. BIRC3 is located on chromosome 11 in proximity to $A T M$ and $11 \mathrm{q}$ deletions include the BIRC3 locus in approximately $80 \%$ of cases. ${ }^{1}$ In addition, BIRC3 can be affected by mutations, mainly nonsense and frameshift variants, with an incidence of $3-5 \%$ in untreated patients, making such mutations rare in comparison to TP53, NOTCH1, SF3B1 or ATM defects..$^{1-5}$ However, as the frequency in fludarabine-refractory cohorts is higher, BIRC3 abnormalities were discussed to define a high-risk group of CLL patients. Indeed BIRC3 did turn out to have an adverse prognostic impact in some chemotherapy-treated CLL cohorts. ${ }^{1,6}$ Furthermore, BIRC3 abnormalities are associat- ed with worse outcome in other lymphomas, acute lymphoblastic leukemia and solid tumors, including brain tumors in which BIRC3 is reported to induce malignant transformation of low-grade gliomas to glioblastomas. ${ }^{6-8}$

While some studies have provided evidence of a clinical impact of mutated BIRC3 and others have not, the functional implications of BIRC3 deletion or mutation are partially unexplored. BIRC3 induces proteasomal degradation of MAP3K14, which is the major driver of noncanonical nuclear factor kappaB (NFкB) activation. Therefore, disrupted BIRC3 could result in a ligand-independent activation of the constitutive NFKB pathway, inducing cell proliferation and survival. ${ }^{7,8}$ At this point Diop and colleagues began their functional characterization of BIRC3 mutations in CLL as described in this issue of Haematologica. ${ }^{9}$ First they confirmed the importance of

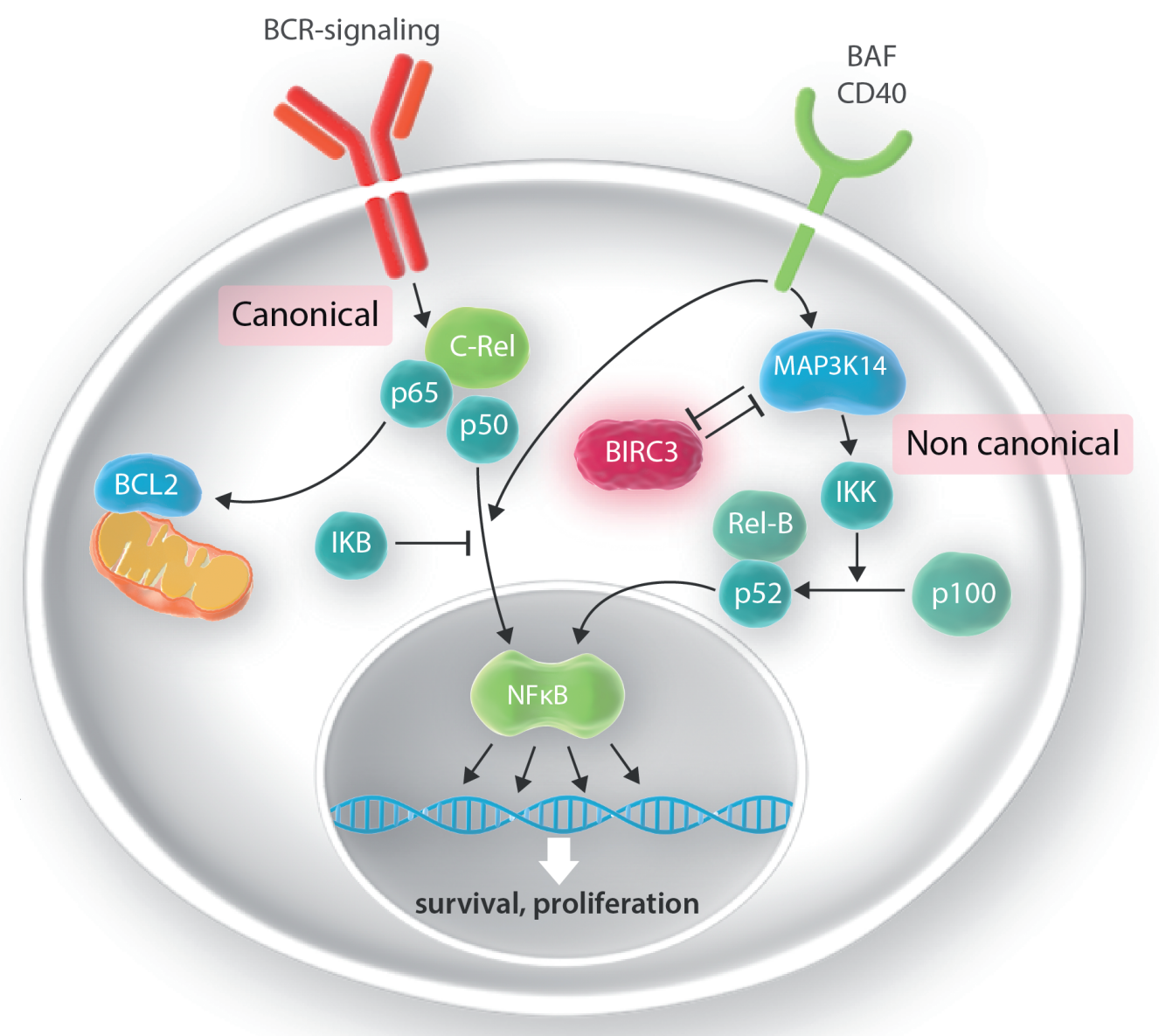

Figure 1. The canonical and non-canonical nuclear factor kappaB pathways. In the canonical nuclear factor kappaB (NFKB) pathway, activation of the B-cell receptor (BCR) results in a shift of the transcription factors c-Rel, p65 and p50 to the nucleus if not sequestered by IKB. In the non-anonical pathway, activation of MAP3K14 results in proteolytic cleavage of p100 to p52, which as a heterodimer with Rel-B serves as a transcription regulator. BIRC3, which is part of the negative regulatory complex, induces proteosomal degradation of MAP3K14, the major driver of activation of the non-canonical NFKB pathway. 
the BIRC3-Map3K14 interaction for activation of the non-canonical NFkB pathway. Silencing Map3K14 by short hairpin RNA decreased the levels of NFKB, which was followed by reduced viability of BIRC3-mutated cells. MAP3K14 could, therefore, be a potential drug target to overcome mutant BIRC3-induced cell proliferation. Next Diop and colleagues evaluated the vulnerability of BIRC3-mutated cell lines and primary CLL cells to fludarabine. Viability assays under fludarabine treatment confirmed that BIRC3-mutated samples had a higher viability than BIRC3 wildtype ones, although still lower than that of TP53-mutated samples after $48 \mathrm{~h}$. Therefore, refractoriness to fludarabine, which has been consistently assigned to TP53 defects in previous clinical and biological studies, is also found to a lesser extent in BIRC3mutated cells. This translates into a significantly shorter progression-free survival of patients with BIRC3-mutated CLL receiving therapy with fludarabine, cyclophosphamide and rituximab (FCR), as found by the authors in a cohort of 287 previously untreated CLL patients.

This effect on outcome may be different with more modern treatment regimens. Although Diop and colleagues provide some evidence that NFKB in BIRC3mutated patients remains active with ibrutinib therapy, there are more downstream targets of Bruton tyrosine kinase (BTK), including MEK/ERK and MAPK, which should remain inhibited by ibrutinib. ${ }^{10}$ In general, in vitro cultures are less informative regarding the efficacy of BTK inhibitors for which the microenvironment plays a crucial role. In contrast to ibrutinib, treatment with venetoclax resulted in a similarly low viability of BIRC3-mutated and wildtype primary CLL cells. ${ }^{9}$ This appears rational, as BCL2 is not involved in the non-canonical pathway affected by BIRC3. However, there is also some evidence that BCL2 levels are higher in BIRC3-mutated cases, indicating a greater sensitivity to venetoclax. ${ }^{11}$ Although only limited data on the impact of BIRC3 mutations are available from clinical trials with ibrutinib and venetoclax, an adverse outcome has not been observed, in contrast to del17p/mutated TP53. ${ }^{12,13}$

Despite the comprehensive work by Diop and colleagues some questions remain in addition to the unclear impact on outcome with novel compounds. This include the difference between monoallelic and biallelic defects (i.e., mutations and deletions), which considered together should result in a much higher number of affected patients. Furthermore the role of non-truncating missense variants and mutations outside the C-terminal RINGdomain found predominantly in solid tumors, but also in lymphomas and CLL, remains unclear. Therefore, further studies, in particular in cohorts of patients from prospective trials evaluating biologically targeted agents, are warranted before BIRC3 assessment can be put forward as a routine test in general clinical practice.

\section{References}

1. Rose-Zerilli MJJ, Forster J, Parker H, et al. ATM mutation rather than BIRC3 deletion and/or mutation predicts reduced survival in 11qdeleted chronic lymphocytic leukemia: data from the UK LRF CLL4 trial. Haematologica. 2014;99(4):736-742.

2. Rossi D, Bruscaggin A, Spina V, et al. Mutations of the SF3B1 splicing factor in chronic lymphocytic leukemia: association with progression and fludarabine-refractoriness. Blood. 2011;118(26):6904-6908.

3. Landau DA, Tausch E, Taylor-Weiner AN, et al. Mutations driving CLL and their evolution in progression and relapse. Nature. 2015;526(7574):525-530

4. Nadeu F, Delgado J, Royo C, et al. Clinical impact of clonal and subclonal TP53, SF3B1, BIRC3, NOTCH1, and ATM mutations in chronic lymphocytic leukemia. Blood. 2016;127(17):2122-2130.

5. Baliakas P, Hadzidimitriou A, Sutton L-A, et al. Recurrent mutations refine prognosis in chronic lymphocytic leukemia. Leukemia. 2015;29(2):329-336.

6. Rossi D, Fangazio M, Rasi S, et al. Disruption of BIRC3 associates with fludarabine chemorefractoriness in TP53 wild-type chronic lymphocytic leukemia. Blood. 2012;119(12):2854-2862.

7. Zarnegar BJ, Wang Y, Mahoney DJ, et al. Noncanonical NF-kappaB activation requires coordinated assembly of a regulatory complex of the adaptors CIAP1, CIAP2, TRAF2 and TRAF3 and the kinase NIK. Nat Immunol. 2008;9(12):1371-1378.

8. Rickert RC, Jellusova J, Miletic AV. Signaling by the tumor necrosis factor receptor superfamily in B-cell biology and disease. Immunol Rev. 2011;244(1):115-133.

9. Diop F, Moia R, Favini C, et al. Biological and clinical implications of BIRC3 mutations in chronic lymphocytic leukemia. Haematologica 2020;105(2):448-456.

10. Cheng S, Ma J, Guo A, et al. BTK inhibition targets in vivo CLL proliferation through its effects on B-cell receptor signaling activity. Leukemia. 2014;28(3):649-657.

11. Asslaber D, Wacht N, Leisch M, et al. BIRC3 expression predicts CLL progression and defines treatment sensitivity via enhanced NF- $\mathrm{\kappa B}$ nuclear translocation. Clin Cancer Res. 2019;25(6):1901-1912.

12. Tausch E, Bahlo J, Robrecht S, et al. Genetic markers and outcome in the CLL14 trial of the GCLLSG comparing front line obinutuzumab plus chlorambucil or venetoclax in patients with comorbidity. Hematol Oncol. 2019;37(S2):84-86.

13. Brown JR, Hillmen P, O'Brien S, et al. Extended follow-up and impact of high-risk prognostic factors from the phase 3 RESONATE study in patients with previously treated CLL/SLL. Leukemia. 2018;32(1):83-91. 